MATHEMATICS OF COMPUTATION

Volume 68, Number 225, January 1999, Pages 321-332

S 0025-5718(99)01036-4

\title{
A CENSUS OF CUSPED HYPERBOLIC 3-MANIFOLDS
}

\author{
PATRICK J. CALLAHAN, MARTIN V. HILDEBRAND, AND JEFFREY R. WEEKS
}

\begin{abstract}
The census provides a basic collection of noncompact hyperbolic 3 -manifolds of finite volume. It contains descriptions of all hyperbolic 3manifolds obtained by gluing the faces of at most seven ideal tetrahedra. Additionally, various geometric and topological invariants are calculated for these manifolds. The findings are summarized and a listing of all manifolds appears in the microfiche supplement.
\end{abstract}

\section{INTRODUCTION}

The classification of 3-dimensional manifolds has been one of the fundamental problems confronting topologists for the last century. In 1982, William Thurston announced his Geometrization Conjecture. Roughly, this conjecture says that all 3 -manifolds can be decomposed in a canonical way into geometric pieces. These geometric pieces are all of the form $X / \Gamma$ for some Riemannian manifold $X$, and some discrete torsion-free subgroup $\Gamma$ of isometries of $X$. Thurston showed that there were only eight such $X$ necessary (see [T1] and [T2] for more details). Of these eight geometries, seven are well understood. In fact the manifolds admitting such geometric structures have been completely classified (see $[\mathrm{S}]$ ). The unresolved case is the class of hyperbolic manifolds, i.e., those manifolds homeomorphic to $H^{3} / \Gamma$ for $\Gamma$ a discrete torsion-free subgroup of isometries. It turns out that in some sense "most" 3-manifolds are hyperbolic, similar to what occurs in the 2dimensional case (see chapter 5 of [T1]). Thus, there is a great amount of interest in the investigation of the structures of hyperbolic 3-manifolds with the hope of discovering some underlying organization which could lead to a classification.

It has been shown [My] that every compact 3-manifold contains a simple closed curve whose complement admits a noncompact hyperbolic structure of finite volume. Furthermore, every noncompact hyperbolic 3-manifold of finite volume can be decomposed into a finite collection of ideal hyperbolic tetrahedra (see [EP]). Therefore, a natural place to start is to enumerate all the possible hyperbolic 3manifolds obtainable from gluing a small number of ideal tetrahedra together. Note that given $n$ ideal tetrahedra, for each set of face pairings there are $6^{2 n}$ possible gluings. Even for $n=6$ there are 97 inequivalent sets of face pairings. Thus, it is impractical even for small $n$ to check all possible gluings. One must find effective ways of eliminating the large numbers of gluings which could not possibly yield hyperbolic manifolds for combinatorial or topological reasons or which have been obtained previously. This process was started in $[\mathrm{HW}]$. Section 4 describes how the enumeration was carried out.

Received by the editor May 26, 1996.

1991 Mathematics Subject Classification. Primary 57-04; Secondary 57M50.

(C)1999 American Mathematical Society 
After the list of all gluings which could possibly yield hyperbolic manifolds had been made, it was then processed by a collection of special purpose computer programs, many of which were based on SnapPea (see [W1]). These programs determined which gluings in fact admitted hyperbolic structures, removed duplicates from the list, and calculated the various invariants described below. For other kinds of computer generated censuses of 3-manifolds see [E], [L], and [MF].

The census provides a large collection of hyperbolic 3-manifolds which can be investigated for typical and atypical properties. The census is also used as a testing ground for hyperbolic 3-manifolds. By enumerating all the possible gluings we get a wide variety of examples: knot and link complements, orientable and nonorientable, singly cusped and multicusped, arithmetic and nonarithmetic manifolds. Of course, by restricting the census to only those manifolds obtained from gluing small numbers of tetrahedra, we are in some sense looking at a special case. On the other hand many questions like "What is the smallest or simplest hyperbolic 3 -manifold with a certain property?" would naturally have candidates among the census manifolds. Furthermore, by considering the census as a whole we could gain some idea of the variety and distribution of the set of all hyperbolic 3-manifolds. Perhaps, even among these small examples, one could glimpse the underlying structure or at least propose provisional classification schemes.

\section{SUMmARY OF RESULTS}

There are 6075 noncompact hyperbolic 3-manifolds which can be obtained from gluing 7 or fewer tetrahedra together. Of these 4815 are orientable and 1260 are nonorientable. More information is summarized in the following tables which are organized by the number of tetrahedra and the cusp type (see section 3 for conventions for describing cusp type).

$\begin{array}{lrrrrrrrr}\text { Orientable: } & & & & & & & \\ \text { cusp type|\# tetrahedra } & 1 & 2 & 3 & 4 & 5 & 6 & 7 & \text { total } \\ 1 & - & 2 & 9 & 52 & 223 & 913 & 3388 & 4587 \\ 2 & - & - & - & 4 & 11 & 48 & 162 & 225 \\ 3 & - & - & - & - & - & 1 & 2 & 3 \\ \text { total } & - & 2 & 9 & 56 & 234 & 962 & 3552 & 4815\end{array}$

$\begin{array}{lrrrrrrrr}\text { Nonorientable: } & & & & & & \\ \text { cusp type|\# tetrahedra } & 1 & 2 & 3 & 4 & 5 & 6 & 7 & \text { total } \\ 1 & 1 & 1 & 5 & 14 & 52 & 171 & 617 & 861 \\ 2 & - & 1 & 2 & 9 & 23 & 68 & 208 & 311 \\ 3 & - & - & - & - & - & 3 & 6 & 9 \\ 4 & - & - & - & - & - & 1 & - & 1 \\ 0,1 & - & - & - & 1 & 1 & 4 & 19 & 25 \\ 0,2 & - & - & - & - & - & 1 & - & 1 \\ 1,1 & - & - & - & 1 & 2 & 8 & 31 & 42 \\ 2,1 & - & - & - & 1 & - & 3 & 6 & 10 \\ \text { total } & 1 & 2 & 7 & 26 & 78 & 259 & 887 & 1260\end{array}$




\section{NOMENClATURE}

It is worthwhile to establish a standardized nomenclature for the manifolds in the census so that they may be referred to with ease. We use a system analogous to that used in the knot and link tables. We sort all the hyperbolic 3-manifolds obtainable by gluing $N$ tetrahedra (but which cannot be made with fewer tetrahedra) in order of increasing volume. We then separate the orientable and nonorientable manifolds and organize them by cusp type. For orientable manifolds we use the notation $M T_{n}^{k}$ for the $n$th orientable manifold made from $T$ tetrahedra on the list with $k$ cusps. As with the link tables, if the manifold has only one cusp we suppress the " $k$ " in the notation. For example the manifold $M 2_{1}$ corresponds to the smallest orientable hyperbolic 3-manifold obtained by gluing two ideal tetrahedra and having one cusp. $M 2_{1}$ happens to be homeomorphic to the complement of the figure eight knot and shares its volume with the so-called "figure eight knot sister" denoted $M 2_{2}$. One of the smallest known hyperbolic links is the Whitehead link which can be decomposed into four ideal tetrahedra. It shows up in the census as $M 4_{1}^{2}$.

The nomenclature for nonorientable manifolds is only slightly more complicated due to the fact that they can have both orientable and nonorientable cusps (the cross sections are tori and Klein bottles, respectively). The basic symbol is the same as for the orientable manifolds, except we use an " $N$ " to indicate nonorientable instead of an " $M$ " for manifold. The symbol $N T_{n}^{c}$ stands for the $n$th nonorientable hyperbolic 3-manifold made from $T$ tetrahedra on the list with cusp type " $c$ ", where " $c$ " is the string " $k, t$ " and $k$ is the number of Klein bottle cusps and $t$ is the number of torus cusps. We also use the convention that we drop the ",0" if there are no orientable cusps and drop the "1,0" if there is exactly one Klein bottle cusp. For example the smallest noncompact hyperbolic 3-manifold (see [A]) is the Gieseking manifold which is obtained by identifying the faces of a single ideal tetrahedron. The Gieseking manifold has exactly one cusp which is nonorientable, hence it is described as $N 1_{1}$. The link $6_{2}^{2}$ double covers a nonorientable manifold with two nonorientable cusps, this manifold is described as $N 2_{1}^{2}$. One of the smallest known nonorientable manifolds with an orientable cusp also happens to have two nonorientable cusps. It appears in the census as $N 4_{1}^{2,1}$ and happens to be the unique 3-cusped manifold which can be made with four or fewer tetrahedra (see $[\mathrm{AS}])$.

\section{Rehydrating the Census manifolds}

Each manifold in the census is obtained from gluing together at most 7 tetrahedra. Since Mostow's Rigidity Theorem implies that there is a unique hyperbolic structure, it suffices to provide the combinatorial gluing pattern, i.e., a set of tetrahedra with labelings to indicate identifications. This gluing pattern is encoded in a string of letters called the "dehydrated manifold". The basic ideas behind this encoding are due to Thurston. We will describe below how the gluing instructions can be recovered by "rehydrating". Once the manifold is rehydrated, the actual hyperbolic structure, i.e., the shapes of the ideal tetrahedra can be found by solving the holonomy equations as described in [T1].

We first describe the general procedure and then give the details for two examples. The rehydrating procedure may be more easily assimilated by following one of the examples given below while reading through the general description. 
Each dehydrated manifold consists of a string of letters. The first letter tells how many tetrahedra are in the manifold, offset from "a": "b" means 1 tetrahedron, "c" means 2, "h" means 7 tetrahedra, etc. Given $N$ tetrahedra, $2 N$ face pairings are necessary. We will number these face pairings 1 through $2 N$. We will recover the face pairing descriptions sequentially.

Label a tetrahedron " 0 " and label its vertices "0" through " 3 ". Label the faces "0" through " 3 " by naming each face after the vertex opposite it. Now, since there are $N$ tetrahedra, $N-1$ of the $2 N$ face pairings will involve a "new" tetrahedron, i.e., a tetrahedron which has not been used yet. The information about when new tetrahedra are added is encoded in the next $2[(N+3) / 4]$ characters (where $[\mathrm{X}]$ is the greatest integer less than or equal to $\mathrm{X}$ ). These characters are read by taking pairs $\mathrm{XY}$ and translating as a number $16 \mathrm{X}+\mathrm{Y}$ which will be between 0 and 255 . The $\mathrm{X}$ and $\mathrm{Y}$ are the just the number of the alphabet offset from "a", i.e., "a" $=0$, "b" $=1$, etc. The numerical value of the pair XY is then written in binary. This binary number read right to left gives the information for the first 8 face pairings: "0" means glue to an old tetrahedron, " 1 " means glue a new tetrahedron. If $N>4$, then the next pair of characters is interpreted in the same way to describe the face pairings numbered 9 though 16 .

To describe a face pairing we must indicate one face of one tetrahedron and one face of another tetrahedron and tell how these two faces are identified. To do this we form a queue of "open" faces. We start with face 0 of tetrahedron 0 and determine where it is glued. Then we move to face 1 of tetrahedron 0 (unless it has already been glued, in which case we move on to face 2). After we have dealt with face 3 of tetrahedron 0 , we move on to face 0 of tetrahedron 1 (or whichever face is still open, in order). To move along the queue we need to know where the indicated face is glued and how the face is glued. First, to determine which tetrahedron the face is glued to depends on whether this particular face pairing involves a new or old tetrahedron as determined above. Exactly $N-1$ will involve new tetrahedra.

If the gluing involves a new tetrahedron, then we give this new tetrahedron the smallest number which has not been used. Then we glue this to the open face we are considering via the identity map, e.g., if we are working with face 1 , then we label the glued face of the new tetrahedron 1 and we label the vertices 0,2 , and 3 to match up with the corresponding vertices of the old tetrahedron. Then we label the remaining faces of the new tetrahedron from their opposite vertices. Thus, the new tetrahedron gets an induced labeling and becomes one of the old tetrahedra.

If the gluing involves an old tetrahedron, then we need to know to which face and to which tetrahedron the current face is glued. Since there will be exactly $N+1$ gluings which involve old tetrahedra, the last $N+1$ characters in the string encode how the face is glued. The second from last $N+1$ characters encode to the tetrahedron to which the face is being glued. Reading from left to right these characters will be the letter representing a number offset from "a", i.e., "a" means "glue to tetrahedron 0", "b" means "glue to tetrahedron 1", etc. Once the tetrahedron is known, it suffices to give a description of the face gluing. These can be denoted by a permutation on the symbols " 0 " through " 3 ". If we write ABCD as the permutation which sends 3 to $\mathrm{A}, 2$ to $\mathrm{B}, 1$ to $\mathrm{C}$, and 0 to $\mathrm{D}$, then we can sort the 24 permutations in numerical order and associate them with the letters "a" through "x". For example: "a" would be the permutation 0123, "b" = 0132, "c" $=0213, \ldots$, and "x" $=3210$. 
The permutation encodes the gluing by indicating which face will be glued to the current face under consideration; if this face is $\mathrm{X}$ then the permutation sends $\mathrm{X}$ to the number of the face of the old tetrahedron to which it is being glued. The remaining three numbers in the permutation tell which vertices are glued together. For example, if we are currently working with face 2 , and the permutation is "b" = 0132, then face 2 is glued to face 1 , and the vertices 3,1 , and 0 of face 2 are glued to the vertices 0,3 , and 2 of face 1 , respectively. Recall that all faces and vertices of old tetrahedra have already been given labels.

Example: The Gieseking manifold: $N 1_{1}$. The dehydrated description of the Gieseking manifold is "baaaade". So to rehydrate its gluing pattern we look first at the first character: "b". This means that there is only one tetrahedron being glued together, $N=1$. The next $2[(1+3) / 4]=2$ characters: "aa" tells which of the $2 N$ face pairings involve new tetrahedra. Here "aa" $=0 * 16+0=0$ which in binary is 00000000 . So none of the face pairings involve new tetrahedra (which must be the case since there is only one tetrahedron!). Now we begin the face pairs in sequential order. Beginning with face 0 of tetrahedra 0 , this pairing involves an old tetrahedron, so we look at the first character in the substring consisting of the second from last set of $N+1$ characters: "a" which means we glue to tetrahedron 0 . Now we look at the first character in the substring consisting of the last $N+1$ characters: "d". This represents the permutation 0231 , so face 0 is glued to face 1 , and the vertices $3,2,1$ of face 0 are glued to the vertices $0,2,3$ of face 1 , respectively. Now we go on to the next face pairing (which happens to be the last in this case). We just did face 0 of tetrahedron 0 , thus we move to face 1 of tetrahedron 0 . Since this face has already been glued, we move to face 2, which is still open. This face is glued to tetrahedron 0 , so to determine which face it will be glued to and how it will be glued we look at the next character in the substring consisting of the last $N+1$ characters. This happens to be the last character: "e" which represents the permutation 0312 . This means that face 2 is glued to face 3 , and the vertices $3,1,0$ of face 2 are glued to the vertices $0,1,2$ of face 3 , respectively.

This completes the description, which can be summarized in the following table:

$\begin{array}{llll} & \text { Active face: } & \text { glue to: } & \text { via face map: } \\ 1 & \text { tet } 0 \text { face } 0 & \text { tet } 0 \text { face } 1 & 321 \text { to } 023 \\ 2 & \text { tet } 0 \text { face } 2 & \text { tet } 0 \text { face } 3 & 310 \text { to } 012\end{array}$

Example: A seven tetrahedra example: $M 7_{162}^{2}$. The dehydrated description of $M 7_{162}^{2}$ is "hbpabbcfggfegfkadihgo". The first character "h" tells us that $N=$ 7. The next $2[(7+3) / 4]=4$ characters tell us which face pairings involve new tetrahedra: these characters "bpab" are taken in pairs. The first pair "bp" gives $1^{*} 16+15=31$ which in binary is 00011111 , which means the first five face pairings involve new tetrahedra. The next two characters "ab" give $0 * 16+1=1$, which in binary is 00000001 . This means that the ninth face pairing involves a new tetrahedron. Note that this tells us that six of the face pairings involve a new tetrahedron, which is correct, since there must always be exactly $N-1$ such pairings in a given gluing pattern. To facilitate things, we will call the substring consisting of the next $N+1$ characters "bcfggfeg" the W string (for which tetrahedron will be glued) and the substring consisting of the last $N+1$ characters "fkadihgo" the $\mathrm{H}$ string (for how the tetrahedra are glued). 
We start with face 0 of tetrahedron 0 . It involves a new tetrahedron which we call tetrahedron 1 . We glue these together by the identity map on face 0 . Now we go on to face 1 of tetrahedron 0 . This also involves a new tetrahedron which we call tetrahedron 2 which is glued to face 1 . Thus we label the glued face of tetrahedron 2 as 1 and label the vertices of the face to match those of face 1 of tetrahedron 0 . This induces a labeling on the rest of the faces and vertices of tetrahedron 2 .

We continue on: face 2 of tetrahedron 0 gets a new tetrahedron which we call 3 . Face 3 of tetrahedron 0 gets a new tetrahedron which we call 4 . Now we move on to face 0 of tetrahedron 1 , which has already been glued (to face 0 of tetrahedron 0 ). Thus we go on to face 1 of tetrahedron 1 , which is still open, and we give it a new tetrahedron which we call 5 . We are now on the sixth face pairing. It is the first one which does not involve a new tetrahedron. Thus we look to the first character of W which is "b", which means "glue to tetrahedron 1". The first character of $\mathrm{H}$ is "f", which represents the permutation 0321. Since the face we are considering is face 2 of tetrahedron 1 , the permutation tells us that 2 is sent to 3 . Thus we glue face 2 to face 3 of tetrahedron 1 and the vertices $3,1,0$ of face 2 are glued to the vertices $0,2,1$ of face 3 , respectively. We continue in this fashion until we have completed all 14 face pairings. The results are summarized below.

$\begin{array}{llll} & \text { Active face: } & \text { glue to: } & \text { via face map: } \\ 1 & \text { tet } 0 \text { face } 0 & \text { tet } 1 \text { face } 0 & \text { id } \\ 2 & \text { tet } 0 \text { face } 1 & \text { tet } 2 \text { face } 1 & \text { id } \\ 3 & \text { tet } 0 \text { face } 2 & \text { tet } 3 \text { face } 2 & \text { id } \\ 4 & \text { tet } 0 \text { face } 3 & \text { tet } 4 \text { face } 3 & \text { id } \\ 5 & \text { tet } 1 \text { face } 1 & \text { tet } 5 \text { face } 1 & \text { id } \\ 6 & \text { tet } 1 \text { face } 2 & \text { tet } 1 \text { face } 3 & 310 \text { to } 021 \\ 7 & \text { tet } 2 \text { face } 0 & \text { tet } 2 \text { face } 2 & 321 \text { to } 130 \\ 8 & \text { tet } 2 \text { face } 3 & \text { tet } 5 \text { face } 0 & 210 \text { to } 123 \\ 9 & \text { tet } 3 \text { face } 0 & \text { tet } 6 \text { face } 0 & \text { id } \\ 10 & \text { tet } 3 \text { face } 1 & \text { tet } 6 \text { face } 3 & 320 \text { to } 021 \\ 11 & \text { tet } 3 \text { face } 3 & \text { tet } 6 \text { face } 1 & 210 \text { to } 203 \\ 12 & \text { tet } 4 \text { face } 0 & \text { tet } 5 \text { face } 2 & 321 \text { to } 103 \\ 13 & \text { tet } 4 \text { face } 1 & \text { tet } 4 \text { face } 2 & 320 \text { to } 103 \\ 14 & \text { tet } 5 \text { face } 3 & \text { tet } 6 \text { face } 2 & 210 \text { to } 103\end{array}$

\section{DESCRIPTION OF ENUMERATION PROCESS}

The basic outline of the census was described in $[\mathrm{HW}]$, but further work has incorporated some additional refinements. As described in $[\mathrm{HW}]$, the first step was to enumerate all nonisomorphic connected graphs on $n$ vertices, where each vertex has degree 4 and $n$ is the number of tetrahedra. The graphs correspond to pairings of the faces of the tetrahedra. Each vertex of the graph corresponds to a tetrahedron, and each edge of the graph corresponds to a pair of faces glued together. For $n$ being 1 through 7, the number of such graphs is 1, 2, 4, 10, 28, 97 , and 359, respectively. For each pair of faces, there are potentially six different ways to glue the pair. Three of these ways correspond to orientable gluings while the rest correspond to nonorientable gluings. A recursive routine does this, but the recursion stops when certain Euler characteristic or edge class criteria (described in $[\mathrm{HW}]$ ) cannot be met. For instance, if a complete edge class contains one or two 
edges or three edges with distinct tetrahedra surrounding the edge class, then the manifold will be either nonhyperbolic or will have been obtained previously.

An additional technique used in the 7-tetrahedra census is to examine partial cusps. Part way through the gluings, triangular cross-sections of the ideal vertices of the tetrahedra form "partial cusps" which are surfaces with boundaries. The code checks what would happen if the boundaries of the partial cusps were capped off by disks. If the Euler characteristic of any surface resulting from such capping is negative, then no matter how the remaining face pairs are glued, the final manifold is sure to have an end whose cross section is a surface of negative Euler characteristic. In that case, the manifold cannot possibly be hyperbolic. At first, each partial cusp is a triangle corresponding to an ideal vertex of a tetrahedron. Each time we specify the gluings of the tetrahedral faces (including how the vertices along the faces are identified), we glue three pairs of edges in the partial cusps. The gluings of these edges may change the Euler characteristic and number of boundary components of the partial cusp. The program keeps track of these values as well as edges and vertices along the boundary components of each partial cusp. These edges and vertices of the boundary components of the partial cusp correspond to unglued faces and edges, respectively, of the tetrahedra.

The program examines various cases to determine the change in Euler characteristic and number of boundary components of a partial cusp. The procedure used to examine these cases is described as follows.

1. If the edges in a pair lie on the same boundary component and the edges are the only two edges in the boundary component, then remove the boundary component, subtract 1 from the number of boundary components, and add either 0 or 1 to the Euler characteristic of the cusp. The number added depends on how the vertices of the boundary components are identified.

2. If the two edges lie on the same boundary component are adjacent and are not the only edges on the boundary component, then subtract 0 or 1 from the Euler characteristic and leave the number of boundary components alone. The number subtracted depends on how the vertices of the boundary components are identified.

3. If the two edges lie on the same boundary component and are not adjacent, then the gluing may split the boundary component into two components. If so, then add 1 to the number of boundary components but subtract 1 from the Euler characteristic. Otherwise, subtract 1 from the Euler characteristic of the cusp but leave the number of boundary components alone.

4. If the two edges lie on different boundary components, then merge the cusps if the components are on different cusps. To find the Euler characteristic and the number of boundary components of a merged partial cusp, add the Euler characteristics of the old cusps and add the number of boundary components of the old cusps. If both boundary components have length 1 , subtract 2 from the number of boundary components and leave the Euler characteristic alone. Otherwise, merge components and subtract 1 from the Euler characteristic and the number of boundary components. 

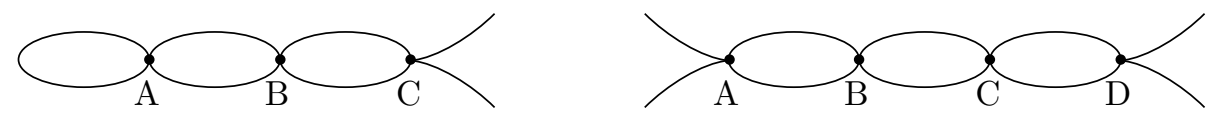

Figure 1

Note that the sum of the Euler characteristic plus the number of boundary components never increases at any step. In the last case, this fact can be seen by noting that this sum is never more than 2 . Thus if this sum is negative at any step before the manifold is finished, we may conclude that the cusp will not be a torus or Klein bottle and that the manifold will not be hyperbolic.

Gluings obtained from this process include all the desired hyperbolic 3-manifolds. A gluing may appear a number of times and may not be a hyperbolic 3-manifold. A terse description which uniquely identifies the gluing helps eliminate the duplication, and SnapPea checks to see which manifolds are hyperbolic. SnapPea also calculates and compares canonical triagulations to remove distinct gluings of isometric manifolds (see [W2] for details).

With programs of this size, bugs naturally creep in. A number of methods were used to test for bugs. Some were described in [HW]. Further checks included tracing through the partial cusp check at the start and then checking by hand. The partial cusp check was also used on a few graphs in the 6-tetrahedra case, and the results agreed with runs for these graphs without this check. Furthermore before we implemented the partial cusp check, we were able to run the census for orientable manifolds obtained from 7 tetrahedra. All tetrahedral gluings which were identified as potentially hyperbolic reappeared when the partial cusp check was added. At the end of a run for each graph, the computer would output a message indicating completion. Thus if the program was no longer running a graph but had not produced this message, we knew that the run had been aborted (perhaps due to a computer failing or being restarted) and that the results could be incomplete unless this graph was run again.

To search only for orientable manifolds, one gives an arbitrary handedness to the first tetrahedron. At each face pairing, if the handedness of both tetrahedra is known, then there are only three ways to glue the pair and keep the manifold orientable. If the handedness of one tetrahedron in the face pairing is known, then there are six possible ways to glue the faces, but this choice determines the handedness of the other tetrahedra. Furthermore, if tetrahedra are glued as in Figure 1, one may, by symmetry, preset the orientation of tetrahedra A, B, and C. In the left portion, the handedness of $\mathrm{C}$ can be changed by interchanging the 2 faces glued with $\mathrm{B}$; this changes the handedness of $\mathrm{B}$. The handedness of $\mathrm{B}$ can also be changed by interchanging the faces glued onto A. This will change the handedness of A, but the handedness of A can also be changed by interchanging the faces glued to each other. A similar argument applies to $\mathrm{A}$ through $\mathrm{C}$ in the right portion provided that tetrahedron $\mathrm{D}$ is not the first tetrahedron. Presetting the orientation was used to speed up the census of orientable manifolds obtained from 7 tetrahedra.

A potential future project for someone attempting to extend the census is to see if one can better exploit symmetries within each graph. Often the same gluings appear a number of times in census runs already performed. 


\section{DESCRIPTION OF THE TABLES}

The 6075 manifolds of the census are organized into several tables. The manifolds made from four or fewer tetrahedra are in the appendixes. The manifolds made from five, six, or seven tetrahedra are in tables included on a microfiche supplement.

Each manifold is listed by name (see section 3 for nomenclature conventions). Various invariants are listed in the table. These are described below.

i. Volume: The primary invariant of hyperbolic 3-manifolds is the volume. The volume is defined with respect to the Riemannian metric of constant curvature -1 . The remarkable thing is that by the Mostow Rigidity Theorem (see [T1]) volume is in fact a topological invariant.

ii. Chern-Simons: Meyerhoff extended the usual Chern-Simons invariant to include noncompact hyperbolic 3-manifolds (see $[\mathrm{M}]$ ). It is well defined modulo $1 / 2$. The Chern-Simons invariant changes sign when the orientation of the manifold changes. Hence, an amphichiral manifold must have a Chern-Simons invariant equal to 0 or $1 / 4$ modulo $1 / 2$ (see $[\mathrm{M}]$ for more information). The Chern-Simons invariant is defined only for orientable manifolds, so this column is not included in the tables of nonorientable manifolds.

iii. Homology: This is the first homology group of the manifold with integer coefficients, i.e., $H_{1}(M)$. The symbol " $\mathrm{Z} / \mathrm{n}$ " denotes the finite cyclic group of order $n$. Note that the rank of $H_{1}(M)$ must be at least equal to the number of cusps.

iv. Symmetry: This is the group of isometries of the manifold. The symbol "Z/n" denotes the finite cyclic group of order $n$. The symbol "Dn" denotes the dihedral group of order $2 n$. If a manifold has a symmetry group which is not a product of cyclic and dihedral groups then only the order of the group is given.

v. $S G$ : This gives the length (to two decimal places) of the shortest closed geodesic in $M$.

vi. $C$ : This describes the chirality or handedness of $M$. If $M$ does not admit an orientation reversing isometry, then $M$ is called chiral and is denoted in the table as "c". If $M$ does admit an orientation reversing isometry, then $M$ is called amphichiral and is denoted in the table as "a". This column is not included in the tables of nonorientable manifolds.

vii. $O C$ : The original census in $[\mathrm{HW}]$ grouped all the manifolds obtained from five or fewer tetrahedra together and sorted them by volume. The manifolds were labeled in order of increasing volume by "mXXX" with XXX ranging from 000 to 414. This column gives the number XXX of the given manifold if it is obtainable from no more than five tetrahedra. For the other manifolds, there were separate numbering schemes depending on the number of tetrahedra and the orientability of the manifold. For manifolds obtainable from six or seven tetrahedra, this column gives the number from the appropriate numbering scheme.

viii. Dehydrated: This gives the dehydrated description of the manifold. Section 4 describes how to reconstruct the manifold from a given string of letters.

\section{ACKNOWLEDGMENTS}

The third named author thanks the Geometry Center (NSF Science and Technology Research Center for Computation and Visualization of Geometric Structures) for its support. 
Appendix A: Orientable Census manifolds with at most 4 tetrahedra

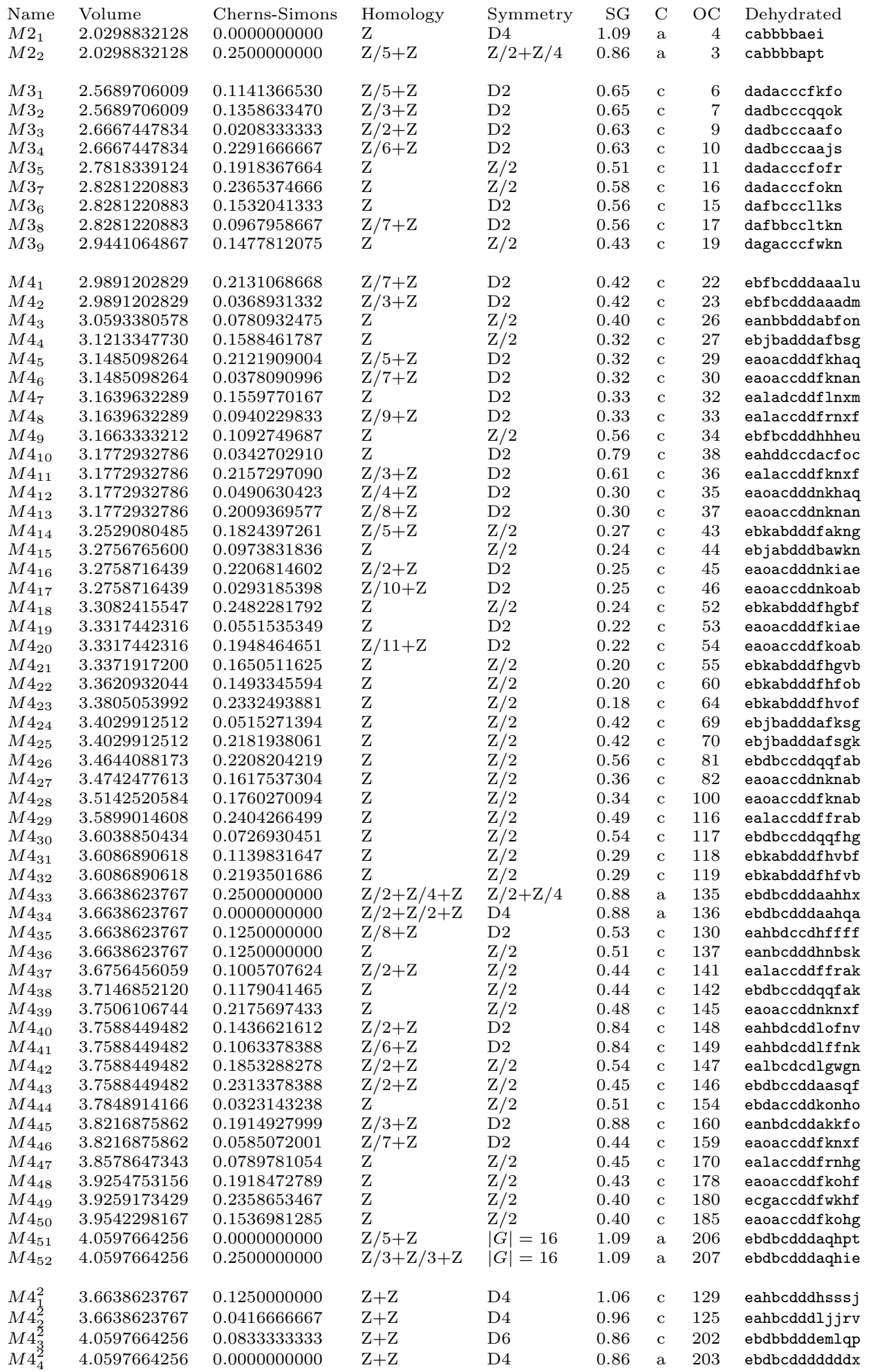


Appendix B: Nonorientable CENSUS MANIFOLdS WITH AT MOST 4 TETRAHEDRA

\begin{tabular}{|c|c|c|c|c|c|c|}
\hline Name & Volume & Homology & Symmetry & SG & $\mathrm{OC}$ & Dehydrated \\
\hline$N 1_{1}$ & 1.0149416064 & $\mathrm{Z}$ & $\mathrm{Z} / 2$ & 1.09 & 0 & baaaade \\
\hline$N 2_{1}$ & 1.8319311884 & $\mathrm{Z} / 2+\mathrm{Z}$ & $\mathrm{Z} / 2$ & 0.88 & 1 & cabbbbabw \\
\hline$N 2_{1}^{2}$ & 2.0298832128 & $\mathrm{Z} / 2+\mathrm{Z}$ & $\mathrm{Z} / 2$ & 0.86 & 2 & cabbbbcdw \\
\hline$N 3_{1}$ & 2.4069095931 & $\mathrm{Z} / 3+\mathrm{Z}$ & $\mathrm{Z} / 2$ & 0.67 & 5 & dafbcccaadl \\
\hline$N 3_{2}$ & 2.6193420504 & $\mathrm{Z}$ & $\mathrm{Z} / 2$ & 0.60 & 8 & dadcbccdjkd \\
\hline$N 3_{3}$ & 2.7868045564 & $\mathrm{Z}$ & $\mathrm{Z} / 2$ & 0.73 & 12 & dadbcccbbcv \\
\hline$N 3_{4}$ & 2.8465105456 & $\mathrm{Z}$ & $\mathrm{Z} / 2$ & 1.28 & 18 & dadbcceccdm \\
\hline$N 3_{5}$ & 3.0448248192 & $\mathrm{Z} / 2+\mathrm{Z} / 2+\mathrm{Z}$ & $\mathrm{Z} / 6$ & 1.09 & 25 & dadbcccaqhx \\
\hline$N 3_{1}^{2}$ & 2.7868045564 & $\mathrm{Z} / 4+\mathrm{Z}$ & $\mathrm{Z} / 2$ & 0.50 & 13 & dadbbcccsbb \\
\hline$N 3_{2}^{2}$ & 2.9539817021 & $\mathrm{Z} / 2+\mathrm{Z}$ & $\mathrm{Z} / 2$ & 0.42 & 20 & dagacccfekl \\
\hline$N 4_{1}$ & 2.7868045564 & $\mathrm{Z} / 4+\mathrm{Z}$ & $\mathrm{Z} / 2$ & 0.50 & 14 & ebdbcdddaaaf $j$ \\
\hline$N 4_{2}$ & 2.9563855471 & $\mathrm{Z} / 3+\mathrm{Z}$ & $\mathrm{Z} / 2$ & 0.41 & 21 & eahbdddcrnenf \\
\hline $\mathrm{N}_{3}$ & 3.1545451962 & $\mathrm{Z}$ & $\mathrm{Z} / 2$ & 0.31 & 31 & eaoacdddnkgaf \\
\hline $\mathrm{N}_{4}$ & 3.1956957274 & $\mathrm{Z} / 2+\mathrm{Z}$ & $\mathrm{Z} / 2$ & 0.28 & 41 & eaoacdddfkgaf \\
\hline$N 4_{5}$ & 3.4853372924 & $\mathrm{Z}$ & $\mathrm{Z} / 2$ & 0.82 & 91 & ealbdcddkfrfh \\
\hline$N 4_{6}$ & 3.6638623767 & $\mathrm{Z} / 2+\mathrm{Z}$ & $\mathrm{Z} / 2$ & 0.96 & 133 & eahbdcddnxxxk \\
\hline$N 4_{7}$ & 3.6638623767 & $\mathrm{Z} / 4+\mathrm{Z}$ & $\mathrm{Z} / 2$ & 0.88 & 132 & ebdbcdddaabbx \\
\hline$N 4_{8}$ & 3.6638623767 & $\mathrm{Z}$ & $\mathrm{Z} / 2$ & 0.69 & 138 & ealbcdddkhdwh \\
\hline$N 4_{9}$ & 3.7775073841 & $\mathrm{Z} / 2+\mathrm{Z}$ & $\mathrm{Z} / 2$ & 0.75 & 153 & ebdbcdddaajfa \\
\hline$N 4_{10}$ & 3.7775073841 & $\mathrm{Z} / 6+\mathrm{Z}$ & $\mathrm{Z} / 2$ & 0.75 & 152 & ebdbcdddaahkc \\
\hline$N 4_{11}$ & 3.7940901118 & $\mathrm{Z}$ & $\mathrm{Z} / 2$ & 0.79 & 156 & eanadcddlnbtq \\
\hline$N 4_{12}$ & 3.8182598148 & $\mathrm{Z} / 2+\mathrm{Z}$ & $\mathrm{Z} / 2$ & 0.76 & 158 & eahbdcddlafnu \\
\hline$N 4_{13}$ & 3.8963450465 & $\mathrm{Z}$ & $\mathrm{Z} / 2$ & 0.68 & 177 & ealbcdddpndoq \\
\hline$N 4_{14}$ & 3.9696478012 & $\mathrm{Z}$ & $\mathrm{Z} / 2$ & 0.89 & 187 & eahdccddakfhq \\
\hline$N 4_{1}^{2}$ & 3.1268546407 & $\mathrm{Z} / 2+\mathrm{Z}$ & $\mathrm{Z} / 2$ & 0.32 & 28 & ebjbadddafbpd \\
\hline$N 4_{2}^{2}$ & 3.2822525639 & $\mathrm{Z} / 2+\mathrm{Z}$ & $\mathrm{Z} / 2$ & 0.24 & 48 & ebkabdddfadnp \\
\hline$N 4_{3}^{2}$ & 3.3372473279 & $\mathrm{Z} / 2+\mathrm{Z}$ & $\mathrm{Z} / 2$ & 0.20 & 56 & ebkabdddfhgmi \\
\hline$N 4_{4}^{2}$ & 3.3817493792 & $\mathrm{Z} / 4+\mathrm{Z}$ & $\mathrm{Z} / 2$ & 0.17 & 65 & ebkabdddfhiom \\
\hline$N 4_{5}^{2}$ & 3.6638623767 & $\mathrm{Z} / 4+\mathrm{Z}$ & D2 & 1.53 & 126 & ebdbcdddccvca \\
\hline$N 4_{6}^{2}$ & 3.6638623767 & $\mathrm{Z} / 2+\mathrm{Z} / 2+\mathrm{Z}$ & D2 & 1.06 & 134 & eahbcdddhhhhx \\
\hline$N 4_{7}^{2}$ & 3.6638623767 & $\mathrm{Z} / 8+\mathrm{Z}$ & D2 & 1.06 & 127 & ebdbcdddaccax \\
\hline$N 4_{8}^{2}$ & 4.0597664256 & $\mathrm{Z} / 2+\mathrm{Z}$ & $\mathrm{Z} / 2$ & 1.66 & 204 & eahbcdcdidxid \\
\hline$N 4_{9}^{2}$ & 4.0597664256 & $\mathrm{Z} / 2+\mathrm{Z}$ & $\mathrm{Z} / 2$ & 0.86 & 205 & ebdbcdddcemre \\
\hline$N 4_{1}^{0,1}$ & 3.6638623767 & $\mathrm{Z}+\mathrm{Z}$ & D2 & 0.88 & 131 & ebdbcdddbbbbx \\
\hline$N 4_{1}^{1,1}$ & 3.6638623767 & $\mathrm{Z}+\mathrm{Z}$ & D2 & 1.32 & 128 & eahbcdddjsssh \\
\hline$N 4_{1}^{2,1}$ & 3.6638623767 & $\mathrm{Z} / 2+\mathrm{Z}+\mathrm{Z}$ & D4 & 1.76 & 124 & eahbcdddjxxxj \\
\hline
\end{tabular}




\section{REFERENCES}

[A] C. Adams, The non-compact hyperbolic 3-manifold of minimal volume, Proc. Amer. Math. Soc. 100 (1987), 601-606. MR 88m:57018

[AS] C. Adams and W. Sherman, Minimal ideal triangulations of hyperbolic 3-manifolds, Discrete Comput. Geom. 6 (1991), 135-153. MR 91m:57009

[E] J. Emert, private communication.

[EP] D. Epstein and R. Penner, Euclidean decompositions of noncompact hyperbolic manifolds, J. Diff. Geom. 27 (1988), 67-80. MR 89a:57020

[HW] M. Hildebrand and J. Weeks, A computer generated census of cusped hyperbolic 3-manifolds, Computers and Mathematics (E. Kaltofen and S. Watt, eds.), Springer-Verlag, 1989, pp. 5359. MR 90f:57043

[L] S. Lins, Gems, Computers and Attractors for 3-Manifolds, Series on Knots and Everything 5 (1995). MR 97f:57012

[MF] S. Matveev and A. Fomenko, Constant energy surfaces of Hamiltonian systems, enumeration of three-dimensional manifolds in increasing order of complexity, and computation of volumes of closed hyperbolic 3-manifolds, Russian Math. Surveys 43 (1988), 3-24.

[M] R. Meyerhoff, Density of the Chern-Simons invariant for hyperbolic 3-manifolds, Low Dimensional Topology and Kleinian Groups, Cambridge University Press, 1986, pp. 217-239. MR 88k:57033a

[My] R. Myers, Simple knots in compact, orientable 3-manifolds, Trans. Amer. Math. Soc. 273 (1982), 75-92. MR 83h:57018

[S] P. Scott, The geometries of 3-manifolds, Bull. London Math. Soc. 15 (1983), 401-487. MR 84m:57009

[T1] W. Thurston, The geometry and topology of 3-manifolds, Princeton Lecture Notes.

[T2] W. Thurston, Three-Dimensional Geometry and Topology, Volume 1 (S. Levy, ed.), Princeton University Press, 1997. MR 97m:57016

[W1] J. Weeks, SnapPea: A computer program for creating and studying hyperbolic 3-manifolds, available by anonymous ftp from geom.umn.edu/pub/software/snappea/.

[W2] J. Weeks, Convex hulls and isometries of cusped hyperbolic 3-manifolds, Topology Appl. 52 (1993), 127-149. MR 95a:57021

Department of Mathematics, University of Texas at Austin, Austin, TX 78712

E-mail address: callahan@math.utexas.edu

Department of Mathematics and Statistics, State University of New York, UniverSity at Albany, Albany, NY 12222

E-mail address: martinhi@math.albany.edu

88 State St., Canton, NY 13617

E-mail address: weeks@geom.umn.edu 